\title{
Fringe-Pattern Demodulation Using a Parametric Method Based on Differential Evolution
}

\author{
J.F. Jimenez ${ }^{1}$, F.J. Cuevas ${ }^{2}$, J.H. Sossa ${ }^{1}$, and L.E. Gomez ${ }^{1}$ \\ ${ }^{1}$ Centro de Investigación en Computación-IPN, Unidad Profesional Adolfo-López Mateos, \\ Av. Juan de Dios Bátiz s/n and M. Othón de Mendizábal, Zacatenco, México, DF. 07738, \\ Mexico \\ ${ }^{2}$ Centro de Investigaciones en Óptica A.C. Loma del Bosque \#115, Col. Lomas del \\ Campestre C.P. 37150, León Gto. México \\ jfvielma@cio.mx, hsossa@cic.ipn.mx, fjcuevas@cio.mx, \\ sgomezb08@sagitario.cic.ipn.mx
}

\begin{abstract}
A parametric method to carry out fringe pattern demodulation by means of Differential Evolution is presented. The phase is approximated by the parametric estimation of an nth-grade polynomial so that no further unwrapping is required. On the other hand, a different parametric function can be chosen according to the prior knowledge of the phase behavior. A differential evolution is codified with the parameters of the function that estimates the phase. The differential evolution evolves until a fitness average threshold is obtained. The method can demodulate noisy fringe patterns and even a one-image closedfringe pattern successfully.
\end{abstract}

Keywords: Phase retrieval; Fringe analysis; Optical metrology; Differential Evolution.

\section{Introduction}

In optical metrology, a fringe pattern (interferogram) can be represented using the following mathematical expression:

$$
I(x, y)=a(x, y)+b(x, y) \times \cos \left(\omega_{x} x+\omega_{y} y+\phi(x, y)+n(x, y)\right)
$$

where $x, y$ are integer values representing indexes of the pixel location in the fringe image, $a(x, y)$ is the background illumination, $b(x, y)$ is the amplitude modulation and is $\phi(x, y)$ the phase term related to the physical quantity being measured. $\omega_{x}$ and $\omega_{y}$ are the angular carrier frequency in directions $x$ and $y$. The term $n(x, y)$ is an additive phase noise. The purpose of any interferometric technique is to determine the phase term, which is related to the physical quantity, being measured. One way to calculate the phase term $\phi(x, y)$ is by using the phase-shifting technique (PST) [15], which needs at least three phase-shifted interferograms. The phase shift among interferograms must be known and experimentally controlled. This technique can be used when mechanical conditions are met throughout the interferometric experiment. 
On the other hand, when the stability conditions mentioned are not covered, there are many techniques to estimate the phase term from a single fringe pattern, such as: the Fourier method [6,7], the Synchronous method [8] and the phase locked loop method (PLL) [9], among others. However, these techniques work well only if the analyzed interferogram has a carrier frequency, a narrow bandwidth and the signal has low noise. Moreover, these methods fail for phase calculation of a closed-fringe pattern. Additionally, the Fourier and Synchronous methods estimate the phase wrapped because of the arctangent function used in the phase calculation, so an additional unwrapping process is required. The unwrapping process is difficult when the fringe pattern includes high amplitude noise, which causes differences greater than $2 \pi$ radians between adjacent pixels [10-12].

Recently, regularization [13-15] and neural networks techniques $[16,17]$ have been used to work with fringe patterns, which contain a narrow bandwidth and noise.

In this work, we propose a technique to determine the phase $\phi(x, y)$, from a fringe pattern with a narrow bandwidth and/or noise, by parametric estimation of a global non-linear function instead of local planes in each site $(x, y)$ as it was proposed in $[13,19]$. Differential Evolution (DE) algorithm is a new heuristic approach mainly having three advantages; Finding the true global minimum regardless of the initial parameter values, fast convergence, and using few control parameters. DE algorithm is a population based algorithm like genetic algorithms using similar operators; crossover, mutation and selection. When a noisy closed fringe pattern is demodulated, neither a low-pass filter nor a thresholoding operator is required. On the other hand, regularization techniques need both of them.

\section{DE Applied to Phase Recovery}

The standard Differential Evolution (DE) algorithm, belonging to the family of Evolutionary Algorithms, was described by Storn and Price [20],[21]. It is based on evolution of a population of vectors, which encode potential solutions to the problem and traverse the fitness landscape by means of genetic operators that are supposed to bias their evolution towards better solutions. DE is a relatively new optimisation technique compared with other more established Evolutionary Algorithms, such as Genetic Algorithms, Evolutionary Strategy, and Genetic Programming [22].

$\mathrm{DE}$ is an optimization algorithm that creates new candidate solutions by combining the parent vector and several other vectors of the same population. A candidate replaces the parent only if it has better fitness [22],[23]. DE uses genetic operators, referred to as mutation, crossover and selection. The role of the genetic operators is to ensure that there is sufficient pressure to obtain even better solutions from good ones (exploitation) and to cover sufficiently the solution space to maximize the probability of discovering the global optimum (exploration).

During the initialization of the algorithm, a population of $N P$ vectors, where $N P$ is the number of vectors, each of dimension $D$ (Which is the number of decision variables in the optimization problem), is randomly generated over the feasible search space.

The fringe demodulation problem is difficult to solve when the level of noise affecting the fringe pattern is elevated, since many solutions are possible even for a 
single noiseless fringe pattern. Besides, the complexity of the problem is increased when a carrier frequency does not exist (closed fringes are presented).

Given that for a closed fringe interferogram there are multiple phase functions for the same pattern, the problem is stated as an ill-posed problem in the Hadamard sense, since a unique solution cannot be obtained [23]. It is clear that image of a fringe pattern $I(x, y)$ will not change if $\phi(x, y)$ in Eq. (1) is replaced with another phase function $\bar{\phi}(x, y)$ given by

$$
\bar{\phi}(x, y)= \begin{cases}-\phi(x, y)+2 \pi & (x, y) \in R \\ \phi(x, y) & (x, y) \notin R\end{cases}
$$

where $R$ is an arbitrary region and $k$ is an integer. In this work, a DE is presented to carry out the optimization process, where a parametric estimation of a non-linear function is proposed to fit the phase of a fringe pattern. Then, DE technique fits a global non-linear function instead of a local plane to each pixel just like it is made in regularization techniques $[13,19]$. The fitting function is chosen depending on the prior knowledge of the demodulation problem as object shape, carrier frequency, pupil size, etc. When no prior information about the shape of $\phi(x, y)$ is known, a polynomial fitting is recommended. In this paper, authors have used a polynomial fitting to show how the method works.

The purpose in any application of DE is to evolve a population of size $N P$ (which codifies NP possible solutions to the problem) using mutation, crossover and selection of each vector, with the goal of optimizing a fitness function adequate to the problem to solve.

In this work, the fitness function $U$, which is used to evaluate the $p$ th vector $a^{p}$ in the population, is given by [18]:

$$
\begin{aligned}
U\left(a^{p}\right)= & \alpha-\sum_{y=1}^{R-1} \sum_{x=1}^{C-1}\left\{\left(I_{N}(x, y)-\cos \left(\omega_{x} x+\omega_{y} y+f\left(a^{p}, x, y\right)\right)^{2}\right.\right. \\
& +\lambda\left[\left(f\left(a^{p}, x, y\right)-f\left(a^{p}, x-1, y\right)\right)^{2}\right. \\
& \left.\left.+\left(f\left(a^{p}, x, y\right)-f\left(a^{p}, x, y-1\right)\right)^{2}\right]\right\} m(x, y),
\end{aligned}
$$

where $x, y$ are integer values representing indexes of the pixel location in the fringe image. Superindex $p$ is an integer index value between 1 and $N P$, which indicates the number of vectors in the population. $I_{N}(x, y)$ is the normalized version of the detected irradiance at point $(x, y)$. The data were normalized in the range $[-1,1] . \omega_{x}$ and $\omega_{y}$ are the angular carrier frequencies in directions $x$ and $y$. The Function $f($.$) is the selected fitting function to carry out the phase approximation. R \times C$ is the image resolution where fringe intensity values are known and $\lambda$ is a smoothness weight factor (it should be clear for the reader that a higher value of parameter $\lambda$ implies a smoother function to be fitted). The binary mask $m(x, y)$ is a field which 
defines the valid area in the fringe pattern. The parameter a can be set to the maximum value of the second term (in negative sum term) at Eq. (3) in the first vector population, which is given by

$$
\begin{aligned}
\alpha= & \max _{p}\left\{\sum _ { y = 1 } ^ { R - 1 } \sum _ { x = 1 } ^ { C - 1 } \left\{\left(I_{N}(x, y)-\cos \left(\omega_{x} x+\omega_{y} y+f\left(a^{p}, x, y\right)\right)^{2}\right.\right.\right. \\
& +\lambda\left[\left(f\left(a^{p}, x, y\right)-f\left(a^{p}, x-1, y\right)\right)^{2}\right. \\
& \left.\left.+\left(f\left(a^{p}, x, y\right)-f\left(a^{p}, x, y-1\right)\right)^{2}\right]\right\} m(x, y),
\end{aligned}
$$

parameter $\alpha$ is used to convert the proposal from minimal to maximal optimization since a fitness function in a DE is considered to be a nonnegative figure of merit and profit [20].

The first term (in negative sum term) at Eq. (3) attempts to keep the local fringe model close to the observed irradiances in least-squares sense. The second term (in negative sum term) at Eq. (3) is a local discrete difference, which enforces the assumption of smoothness and continuity of the detected phase.

At the beginning of a DE, a set of random solutions are codified in a vector population of size $N P$. Each vector $a$ is formed by the parameter function vector (possible solution) and chained string such as:

$$
a=\left[a_{0}\left|a_{1}\right| a_{2}|\ldots| a_{n}\right]
$$

Each dimension $a_{i}$ is a random real number in a defined search range $\left(\min \left(a_{i}\right), \max \left(a_{i}\right)\right)$ (the user defined maximum and minimum of $a_{i}$ ). These values can be initialized using prior knowledge (e.g. in the polynomial case, components $x$ and $y$ are related to the interferogram tilt so if a closed fringe is presented, then these values are near 0 ). Every dimension is generated as:

$$
a_{i}=\operatorname{random}\left(\min \left(a_{i}\right), \max \left(a_{i}\right)\right)
$$

Therefore, the population of $\mathrm{DE}$ consists of $N P$ D-dimensional parameter vectors $X_{i, G}$, where $i=1,2, \ldots, N P$, for each generation $G$.

\subsection{Mutation}

In the mutation step, a difference between two randomly selected vectors from the population is calculated. This difference is multiplied by a fixed weighting factor, $F$, and it is added to a third randomly selected vector from the population, generating the mutant vector, $V[1-3]$.

For each target vector $x_{i, G}$, a mutant vector is produced by;

$$
v_{i, G+1}=x_{i, G}+K \cdot\left(x_{r 1, G}-x_{i, G}\right)+F \cdot\left(x_{r 2, G}-x_{r 3, G}\right)
$$


where $i, r_{1}, r_{2}, r_{3} \in\{1,2, \ldots, N P\}$ are randomly chosen and must be different from each other. In Equation (7), $F$ is the scaling factor which has an effect on the difference vector $\left(x_{r^{2}, G}-x_{r^{3}, G}\right), K$ is the combination factor.

\subsection{Crossover}

After mutation, the crossover is performed between the vector $(X)$ and the mutant vector $(V)$ (Figure 1), using the scheme in (8) to yield the trial vector $(U)$. The crossover probability is determined by the crossover constant $(C R)$, and its purpose is to bring in diversity into the original population [24].

The parent vector is mixed with the mutated vector to produce a trial vector $u_{j i, G+1}$

$$
u_{j i, G+1}= \begin{cases}v_{j i, G+1} & \text { if }\left(r n d_{j} \leq C R\right) \text { or } j=r n_{i}, \\ q_{j i, G} & \text { if }\left(r n d_{j}>C R\right) \text { or } j \neq r n_{i},\end{cases}
$$

where $j=1,2, \ldots, D ;$ rnd $_{j} \in[0,1]$ is the random number; $C R$ is crossover constant $\in[0,1]$ and $r n_{i} \in(1,2, \ldots, D)$ is the randomly chosen index, which ensures that $u_{i, G+1}$ gets at least one parameter from $v_{i, G+1}[19]$.

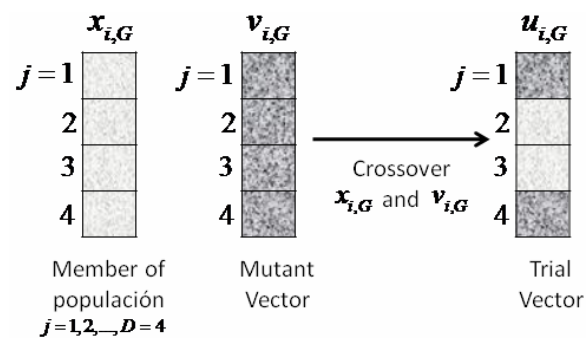

Fig. 1. Illustration of the crossover process for $D=4$

There are different variants that can be used in mutation and crossover, and they are referred to as $\mathrm{DE} / \mathrm{x} / \mathrm{y} / \mathrm{z}$, where $\mathrm{x}$ specifies the vector to be mutated which currently can be "rand" (a randomly chosen population vector) or "best" (vector of the lowest cost from the current population); $y$ is the number of difference vectors used and $z$ denotes the crossover scheme [21].

\subsection{Selection}

In the last step, called selection, the new vectors $(U)$ replace their predecessors if they are closer to the target vector. All solutions in the population have the same 


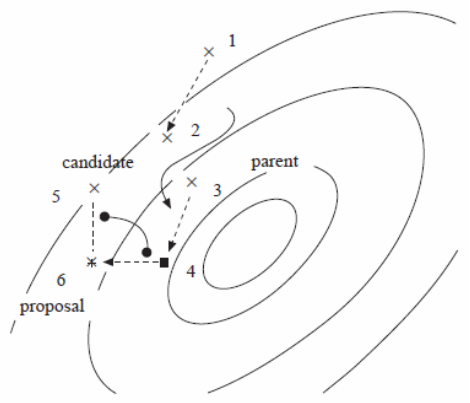

Fig. 2. Obtaining a new proposal in DE

chance of being selected as parents without dependence of their fitness value. The child produced after the mutation and crossover operations is evaluated. Then, the performance of the child vector and its parent is compared and the better one is selected. If the parent is still better, it is retained in the population.

Figure 2 shows DE's process in detail: the difference between two population members $(1,2)$ is added to a third population member (3). The result (4) is subject to the crossover with the candidate for replacement (5) to obtain a proposal (6). The proposal is evaluated and replaces the candidate if it is found to be better.

DE has shown to be effective on a large range of classical optimization problems, and it showed to be more efficient than techniques such as Simulated Annealing and Genetic Algorithms [23],[24]. However, its capability of finding the global optimum is very sensitive to the choice of the control variable F and CR [25]. Consistently with related studies [23],[24],[25], the paper highlights an undesirable behaviour of the algorithm, i.e., the DE does not find the global optimum (value to reach - VTR) when $100 \%$ of the population is trapped in a basin of attraction of a local optimum.

\subsection{DE Convergence}

The DE convergence mainly depends on the population size. It should be clear that if we increase the population size, more vectors will search the global optimum and a best solution will be found in a minor number of iterations, although the processing time can be increased [25].

To stop the DE process, different convergence measures can be employed. In this paper, we have used a relative comparison between the fitness function value of the best vectors in the population and value $a$, which is the maximum possible value to get in Eq. (3). Then, we can establish a relative evaluation of uncertainty to stop the DE as:

$$
\left|\frac{\alpha-U\left(a^{*}\right)}{\alpha}\right| \leq \varepsilon,
$$

where $U\left(a^{*}\right)$ is the fitness function value of the best vectors in the population in the current iteration, and $\varepsilon$ is the relative error tolerance. Additionally, we can stop the process in a specified number of iterations, if Eq. (9) is not satisfied. 


\section{Experiment}

The parametric method using a DE was applied to calculate phase from shadow moiré closed fringe pattern. We used a population size equal to $100, F$ is calculated by values of "F_lower" and "F_higher", in the ranges $[0.1,0.9]$. In each vector, the coded coefficients of a fourth degree polynomial were included. The following polynomial was coded in each vector:

$$
\begin{aligned}
p_{4}(x, y)= & a_{0}+a_{1} x+a_{2} y+a_{3} x^{2}+a_{4} x y+a_{5} y^{2}+a_{6} x^{3}+a_{7} x^{2} y+a_{9} x y^{2} \\
& +a_{9} y^{3}+a_{10} x^{4}+a_{11} x^{3} y+a_{12} x^{2} y^{2}+a_{13} x y^{3}+a_{14} y^{4}
\end{aligned}
$$

so that 15 coefficients were configured in each vector inside population to be evolved.

A low contrasted noisy closed fringe pattern was generated in the computer using the following expression:

$$
I(x, y)=127+63 \cos \left(P_{4}(x, y)+\eta(x, y)\right)
$$

where

$$
\begin{aligned}
p_{4}(x, y)= & -0.7316 x-0.2801 y+0.0065 x^{2}+0.00036 x y-0.0372 y^{2} \\
& +0.00212 x^{3}+0.000272 x^{2} y+0.001 x y^{2}-0.002 y^{3} \\
& +0.000012 x^{4}+0.00015 x^{3} y+0.00023 x^{2} y^{2}+0.00011 x y^{3} \\
& +0.000086 y^{4}
\end{aligned}
$$

and $\eta(x, y)$ is the uniform additive noise in the range [-2radians,2radians]. Additionally, the fringe pattern was generated with a low resolution of $60 \times 60$. In this case, we use a parameter search range of $[-1,1]$. The population of vectors was evolved until the number of iterations and relative error tolerance $\varepsilon$ was 0.05 in Eq. (9). This condition was achieved in $77 \mathrm{~s}$ on a AMD Turion X2-2.4 GHz computer. The fringe pattern and the contour phase field of the computer generated interferogram are shown in Fig. 3.

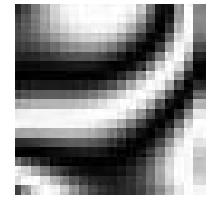

(a)

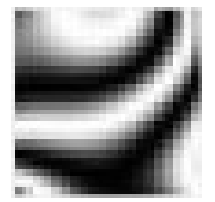

(b)

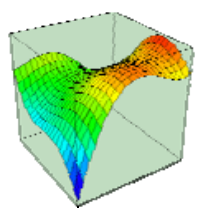

(c)

Fig. 3. (a) Original fringe pattern, (b) phase field obtained by using DE technique and (c) phase obtained in 3D

The DE technique was used to recover the phase from the fringe pattern. The fringe pattern and the phase estimated by DE is shown in Fig. 3. Tests are shown on Table 1, the best vectors for the testers are shown on Table 2, and worst vectors for the testers are shown on Table 3. 
Table 1. Table of parameters of "F_lower" and "F_higher"

\begin{tabular}{cccccccccc}
\hline & 0.1 & 0.2 & 0.3 & 0.4 & 0.5 & 0.6 & 0.7 & 0.8 & 0.9 \\
\hline 0.1 & $2.30 \mathrm{E}-02$ & $4.84 \mathrm{E}-01$ & $7.79 \mathrm{E}-01$ & $6.29 \mathrm{E}-02$ & $1.64 \mathrm{E}-01$ & $2.24 \mathrm{E}-02$ & $2.63 \mathrm{E}-01$ & $3.77 \mathrm{E}-01$ & $7.58 \mathrm{E}-01$ \\
0.2 & $3.60 \mathrm{E}-02$ & $4.80 \mathrm{E}-02$ & $7.19 \mathrm{E}-01$ & $9.19 \mathrm{E}-01$ & $1.21 \mathrm{E}+00$ & $1.52 \mathrm{E}-02$ & $\mathbf{1 . 2 8 E}-\mathbf{0 3}$ & $\mathbf{1 . 4 0 E}-\mathbf{0 4}$ & $\mathbf{9 . 6 3 E}-\mathbf{0 3}$ \\
0.3 & $1.99 \mathrm{E}-02$ & $\mathbf{4 . 3 7 E - 0 2}$ & $\mathbf{1 . 0 8 E}-03$ & $1.32 \mathrm{E}+00$ & $1.83 \mathrm{E}-03$ & $\mathbf{1 . 1 2 E}-\mathbf{0 4}$ & $1.35 \mathrm{E}+00$ & $8.28 \mathrm{E}-03$ & $2.24 \mathrm{E}+00$ \\
0.4 & $7.00 \mathrm{E}-01$ & $1.04 \mathrm{E}+00$ & $1.51 \mathrm{E}+00$ & $3.62 \mathrm{E}-01$ & $\mathbf{1 . 5 8 E}-\mathbf{0 4}$ & $9.44 \mathrm{E}-04$ & $1.61 \mathrm{E}+00$ & $1.92 \mathrm{E}-03$ & $2.90 \mathrm{E}+00$ \\
0.5 & $\mathbf{1 . 0 3 E}-03$ & $2.81 \mathrm{E}-01$ & $1.47 \mathrm{E}+00$ & $1.67 \mathrm{E}+00$ & $3.54 \mathrm{E}-04$ & $1.76 \mathrm{E}+00$ & $2.12 \mathrm{E}+00$ & $1.92 \mathrm{E}+00$ & $1.94 \mathrm{E}+00$ \\
0.6 & $7.88 \mathrm{E}-01$ & $2.02 \mathrm{E}-01$ & $1.44 \mathrm{E}+00$ & $1.35 \mathrm{E}+00$ & $1.46 \mathrm{E}+00$ & $2.23 \mathrm{E}+00$ & $1.80 \mathrm{E}+00$ & $2.72 \mathrm{E}+00$ & $3.14 \mathrm{E}+00$ \\
0.7 & $3.15 \mathrm{E}-01$ & $1.19 \mathrm{E}+00$ & $1.95 \mathrm{E}+00$ & $1.17 \mathrm{E}+00$ & $1.88 \mathrm{E}+00$ & $2.31 \mathrm{E}+00$ & $2.11 \mathrm{E}+00$ & $3.29 \mathrm{E}+00$ & $2.87 \mathrm{E}+00$ \\
0.8 & $9.20 \mathrm{E}-01$ & $1.74 \mathrm{E}+00$ & $1.31 \mathrm{E}+00$ & $1.91 \mathrm{E}+00$ & $2.27 \mathrm{E}+00$ & $2.02 \mathrm{E}+00$ & $2.11 \mathrm{E}+00$ & $2.77 \mathrm{E}+00$ & $3.79 \mathrm{E}+00$ \\
0.9 & $1.03 \mathrm{E}-03$ & $1.89 \mathrm{E}+00$ & $1.40 \mathrm{E}+00$ & $\mathbf{3 . 0 9 E - 0 3}$ & $2.71 \mathrm{E}+00$ & $3.11 \mathrm{E}+00$ & $2.48 \mathrm{E}+00$ & $2.08 \mathrm{E}+00$ & $3.07 \mathrm{E}+00$ \\
\hline
\end{tabular}

Table 2. Shows of the best vectors

\begin{tabular}{|c|c|c|c|c|c|c|c|c|c|}
\hline F_lower & 0.1 & 0.2 & 0.3 & 0.4 & 0.5 & 0.6 & 0.7 & 0.8 & 0.9 \\
\hline F_higher & 0.5 & 0.3 & 0.3 & 0.9 & 0.4 & 0.3 & 0.2 & 0.2 & 0.2 \\
\hline Error & $1.03 \mathrm{E}-03$ & 4.37E-02 & $1.08 \mathrm{E}-03$ & $3.09 \mathrm{E}-03$ & $1.58 \mathrm{E}-04$ & 1.12E-04 & $1.28 \mathrm{E}-03$ & $1.40 \mathrm{E}-04$ & $9.63 \mathrm{E}-03$ \\
\hline & & & & & & & & & \\
\hline
\end{tabular}

Table 3. Shows of the worst vectors

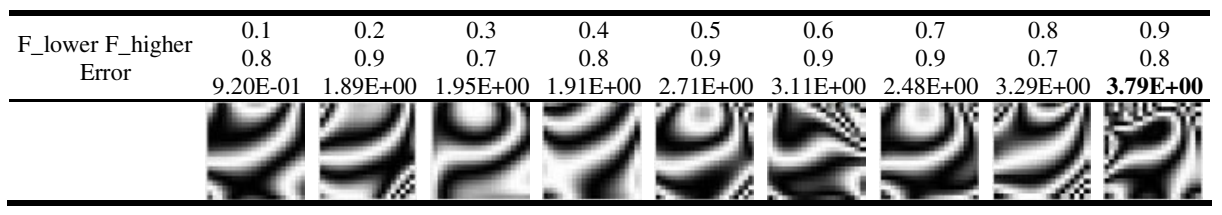

The phases: original, best vector, as worst vector, is shows in the Fig. 4.

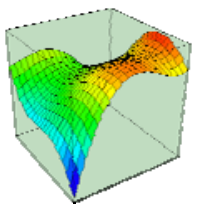

(b)

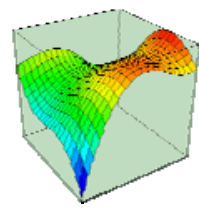

(b)

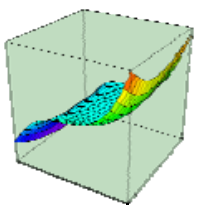

(c)

Fig. 4. Phases: (a) Original, (b) best vector of DE technique and (c) worst vector of DE technique.

\section{Conclusions}

A DE was applied to recover the modulating phase from closed and noisy fringe patterns. A fitness function, which considers the prior knowledge of the object being tested, is established to approximate the phase data. In this work a fourth degree polynomial was used to fit the phase. 
A population of vectors was generated to carry out the optimization process. Each vector was formed by a codified string of polynomial coefficients. Then, the population of vectors was evolved using $\mathrm{CR}, \mathrm{F}$, and $\mathrm{K}$.

The DE technique works successfully where other techniques fail (Synchronous and Fourier methods). This is the case when a noisy, wide bandwidth and/or closed fringe pattern is demodulated. Regularization techniques can be used in these cases but DE technique has the advantage that the cost function does not depend upon the existence of derivatives and restrictive requirements of continuity (gradient descent methods). Since the DE works with a population of possible solutions instead of a single solution, it avoids falling in a local optimum. Additionally, no filters and no thresholding operators were required, in contrast with the fringe-follower regularized phase tracker technique.

The DE has the advantage that if the user knows prior knowledge of the object shape, then a better suited fitting parametric function can be used instead of a general polynomial function. Additionally, due to the fact that the DE technique gets the parameters of the fitting function, it can be used to interpolate sub-pixel values and to increase the original phase resolution or interpolate where fringes do not exist or are not valid. A drawback is the selection of the optimal initial DE parameters (such as population size, F, K) that can increase the convergence speed.

Acknowledgements. We wish to thank the Centro de Investigación en Computación of the I.P.N. by the support to accomplish this project as well as the Centro de Investigaciones en Optica during the image recollections and tests. J. Vielma and L. Gomez thanks CONACYT by the scholarship received to complete his doctoral studies. H. Sossa thanks the SIP-IPN under grant 20091421 for the support. H. Sossa also thanks CINVESTAV-GDL for the support to do a sabbatical stay from December 1, 2009 to May 31, 2010. Authors thank the European Union, the European Commission and CONACYT for the economical support. This paper has been prepared by economical support of the European Commission under grant FONCICYT 93829. The content of this paper is an exclusive responsibility of the CIC-IPN and it cannot be considered that it reflects the position of the European Union. Finally, authors thank the reviewers for their comments for the improvement of this paper.

\section{References}

1. Martín, F., et al.: New advances in Automatic Reading of VLP's. In: Proc. SPC-2000 (IASTED), Marbella, España, pp. 126-131 (2000)

2. Malacara, D., Servin, M., Malacara, Z.: Interferogram Analysis for Optical Testing. Marcel Dekker, New York (1998)

3. Malacara, D.: Optical Shop Testing. Wiley, New York (1992)

4. Creath, K.: In: Wolf, E. (ed.) Progress in Optics, vol. 26, p. 350. Elsevier, Amsterdam (1988)

5. Creath, K.: In: Robinson, D., Reid, G.T. (eds.) Interferogram Analysis, p. 94. IOP Publishing, London (1993)

6. Takeda, M., Ina, H., Kobayashi, S.: Fourier-transform method of fringe-pattern analysis for computer-based topography and interferometry. Journal of Optical Soc. of America 72, 156-160 (1981) 
7. Su, X., Chen, W.: Fourier transform profilometry: a review. Optics and Lasers in Engineering 35(5), 263-284 (2001)

8. Womack, K.H.: Interferometric phase measurement using spatial synchronous detection. Opt. Eng. 23, 391-395 (1984)

9. Servin, M., Rodriguez-Vera, R.: Two dimensional phase locked loop demodulation of interferograms. Journal of Modern Optics 40(11), 2087-2094 (1993)

10. Ghiglia, D.C., Romero, L.A.: Robust two-dimensional weighted and unweighted phase unwrapping that uses fast transforms and iterative methods. Journal of the Optical Society of America A 11(1), 107-117 (1994)

11. Su, X., Xue, L.: Phase unwrapping algorithm based on fringe frequency analysis in Fourier-transform profilometry. Optical Engineering 40, 637-643 (2001)

12. Servin, M., Cuevas, F.J., Malacara, D., Marroquin, J.L., Rodriguez-Vera, R.: Phase unwrapping through demodulation by use of the regularized phase-tracking technique. Applied Optics 38(10), 1934-1941 (1999)

13. Servin, M., Marroquin, J.L., Cuevas, F.J.: Demodulation of a single interferogram by use a two-dimensional regularized phase-tracking technique. Applied Optics 36(19), 4540-4548 (1997)

14. Villa, J., Servin, M.: Robust profilometer for the measurement of 3-D object shapes based on a regularized phase tracker. Optics and Lasers in Engineering 31(4), 279-288 (1999)

15. Quiroga, J.A., Gonzalez-Cano, A.: With a Regularized Phase-Tracking Technique. Applied Optics 39(17), 2931-2940 (2000)

16. Cuevas, F.J., Servin, M., Stavroudis, O.N., Rodriguez-Vera, R.: Multi-Layer neural network applied to phase and depth recovery from fringe patterns. Optics Communications 181(4-6), 239-259 (2000)

17. Cuevas, F.J., Servin, M., Rodriguez-Vera, R.: Depth object recovery using radial Basis Functions. Optics Communications 163(4-6), 270-277 (1999)

18. Cuevas, F.J., Sossa, J.H., Servin, M.: A parametric method applied to phase recovery from a fringe pattern based on a genetic algorithm. Optics Communications, Vol 203(3-6), 213223 (2002)

19. Servin, M., Marroquin, J.L., Cuevas, F.J.: Fringe-follower regularized phase tracker for demodulation of closed-fringe interferograms. Journal of the Optical Society of America A 18(3), 689-695 (2001)

20. Price, K.V., Storn, R.M., Lampinen, J.A.: Differential Evolution - A Practical Approach to Global Optimization. Springer, Heidelberg (2005)

21. Storn, R., Price, K.: Differential Evolution - A Simple and Efficient Heuristic for Global Optimization over Continuous Spaces. Journal of Global Optimization 11(4), 341-359 (1997)

22. Li, X.: Efficient Differential Evolution using Speciation for Multimodal Function Optmization. In: Genetic and Evolutionary Computation Conference. Proceedings of the conference on Genetic and evolutionary computation, Washington, D.C., USA (2005)

23. Robic, T., Filipic, B.: DEMO: Differential Evolution for Multiobjective. In: Proceedings of the 3rd International Conference on Evolutionary MultiCriterion Optimization (EMO), pp. 520-533 (2005)

24. Roger, L.S., Tan, M.S., Rangaiah, G.P.: Global Optimization of Benchmark and Phase Equilibrium Problems Using Differential Evolution. National University of Singapore, Singapore (2006)

25. Gamperle, R., Müller, S.D., Koumoutsakos, P.: A Parameter Study for Differential Evolution. In: Proceedings WSEAS International Conference on Advances in Intelligent Systems, Fuzzy Systems, Evolutionary Computation, pp. 293-298 (2002) 